\title{
Modified Dual Segment Rectangular Dielectric Resonator Antenna Terminated in a Bio-Medium
}

\author{
Ravi Kumar Gangwar ${ }^{1}$, Surya Pal Singh ${ }^{1}$, Devendra Kumar ${ }^{2}$ \\ ${ }^{1}$ Department of Electronics Engineering, Institute of Technology, Banaras Hindu University, Varanasi, India \\ ${ }^{2}$ Department of Ceramic Engineering, Institute of Technology, Banaras Hindu University, Varanasi, India \\ E-mail: \{ravi.gangwar.ece07, spsingh.ece, devendra.cer\}@itbhu.ac.in \\ Received January 27, 2011; revised February 23, 2011; accepted March 15, 2011
}

\begin{abstract}
In this paper, the simulation studies of the radiation characteristics of modified dual segment rectangular dielectric resonator antenna (DSRDRA) in free space and in presence of bio-medium alongwith the absorbed power distribution in a homogenous bio-medium (muscle layer) in direct contact with the proposed antenna are reported at different WiMAX band frequencies. The specific absorption rate (SAR) distributions in muscle layer for different antenna-to-muscle layer spacings are also presented at resonant frequency of antenna in WiMAX bands. The results of return loss, input VSWR and radiation characteristics of proposed antenna in presence of bio-medium are also compared with the results for free space. The simulation study has been carried out using CST Microwave Studio software.
\end{abstract}

Keywords: Modified DSRDRA, SAR, WiMAX Band, Radiation Pattern

\section{Introduction}

The proliferation of wireless portable devices and greatly increased amount of electromagnetic radiation in the environment from these devices are making the public aware of the possible health risks of wireless portable devices. Although the most common fear in people's minds is the risk of contracting serious diseases including brain cancer due to electromagnetic radiation from these devices in the long term. So investigations on wireless portable devices in the vicinity of a human body are important from the technical point of view [1]. The human body, being lossy, absorbs certain amount of electromagnetic radiation generated from wireless portable device situated in its vicinity. Therefore, it is of interest to evaluate the power absorbed/specific absorption rate (SAR) distribution in the body tissues due to wireless device antenna radiating electromagnetic waves [2]. The electric field induced and hence SAR within the human body depends on several factors including the amplitude, frequency and polarization of the electromagnetic wave, and the shape, size and electrical characteristics of the body tissues. As strong interrelation exists among different parameters of dielectric antenna including its size, configuration, dielectric constant, bandwidth, near field distribution and far field pattern; these parameters will affect specific absorption rate (SAR) distribution in a bio-medium located close to the antenna.

The antenna requirements for wireless portable devices are that the antenna be small, have an omni-directional radiation pattern, and have a wide bandwidth capable of operating in that band. Dielectric Resonator Antennas (DRAs) are considered as one of the most suitable portable device antennas due to their low profile, high radiation efficiency, large bandwidth, flexible feed arrangement, wide range of material dielectric constants, ease of excitation, easily controlled characteristics and ease of integration with other active or passive microwave integrated circuit (MIC) components [3-7]. The techniques used to improve the bandwidth of the DRAs include changing the aspect ratio of DRA, employing multisegments and stacked DRAs and by varying the dielectric constant of DRA material. For wider-band applications, DRAs having lower dielectric constant values are preferred. This results in week coupling. Multi-segment DRAs can be used to overcome this problem [8-9].

Only a few studies are reported in the literature on SAR distribution in bio-tissues due to Dielectric Resonator Antennas (DRAs). The FDTD method has been applied for computing SAR distribution inside the human head and the effects of the human proximities including, 
the head, the hand, and the user's glasses on the antenna performance have been analyzed [10]. Off-centre ring DRA has been designed and also used for evaluation of SAR distribution in human head [11]. The performance of rectangular DRA (RDRA) in close proximity with the user's body was studied with the help of a user's hand model [12].

In this paper, simulation studies of the SAR distributions in a homogeneous bio-medium (muscle layer) close to modified DSRDRA, which is fed by a $50 \Omega$ coaxial probe for WiMAX band $(3.2 \mathrm{GHz}$ and $5.0 \mathrm{GHz}$ ), are presented. The radiation characteristics of the proposed antenna and also the SAR distributions in a homogeneous bio-medium (muscle layer) for different antenna-to-bio-medium separations of $0 \mathrm{~cm}, 5 \mathrm{~cm}, 10$ $\mathrm{cm}$ and $20 \mathrm{~cm}$ at resonant frequency of antenna are studied through simulation using CST Microwave Studio software. The simulation results for SAR distribution in the muscle layer are obtained for antenna input power of $0.2 \mathrm{~W}$ and the effect of reducing input power from 0.2 to $0.02 \mathrm{~W}$ on the maximum SAR $(10 \mathrm{~g})$ value is also analyzed. The results of return loss and radiation patterns of proposed antenna in free space are also compared with results of antenna in direct contact with the bio-medium.

\section{Antenna Geometry}

The proposed dual segment RDRA consists of lower segment made from thin teflon sheet with dielectric constant $\varepsilon_{r 1}=2.1$ and an upper segment of alumina block with $\varepsilon_{r 2}=9.8$ as shown in Figure 1. The dual segment DRA is placed on a ground plane of size $40 \times$ $15 \times 4 \mathrm{~mm}^{3}$. The lower and upper segments of the DRA have dimensions of $a \times b \times 1$ and $a \times b \times l_{1}$ respectively. The DRA is excited by a $50 \Omega$ coaxial probe of outer radius $2 \mathrm{~mm}$ and inner radius $0.6 \mathrm{~mm}$. The probe height above the surface of ground plane is found to be 11.6 $\mathrm{mm}$ through simulation using CST Microwave Studio to provide lowest return loss at resonant frequency. A metallic plate of negligible thickness fully covers the side of DRA just opposite the side where feed is located. The design parameters of the antenna are $a=20 \mathrm{~mm}, b$

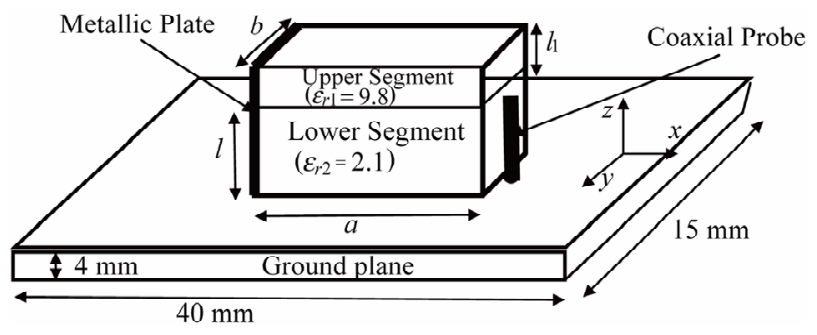

Figure 1. Geometry of modified DSRDRA.
$=12 \mathrm{~mm}, l_{1}=3 \mathrm{~mm}, l=10 \mathrm{~mm}$ and probe height $=11.6$ $\mathrm{mm}[13]$.

\section{Results and Discussion}

\subsection{Return Loss and Input VSWR Characteristics}

The return loss and input impedance versus frequency characteristics for the proposed antenna in free space and in direct contact with bio-medium has been carried out using CST Microwave Studio software. The return loss and input VSWR versus frequency curves of the proposed antenna in free space and in direct contact with bio-medium have been presented in Figures 2 and 3 . From Figure 2 the resonant frequency, operating frequency range and the percentage bandwidth of the proposed RDRA are extracted. The resonant frequency, operating frequency range and the percentage bandwidth of the antenna in free space are found to be $5.156 \mathrm{GHz}$, $3.04-5.65 \mathrm{GHz}$ and $50.62 \%$ respectively.

From Figure 2 it can be seen that the simulated resonant frequency in the presence of phantom muscle is $4.87 \mathrm{GHz}$, which is $286 \mathrm{MHz}$ lower than the resonant frequency of antenna in free space. The frequency de-tuning in the presence of muscle layer may be due to the perturbations in both the resonant mode and near field distribution of the antenna. From Figures 2 and $\mathbf{3}$ it

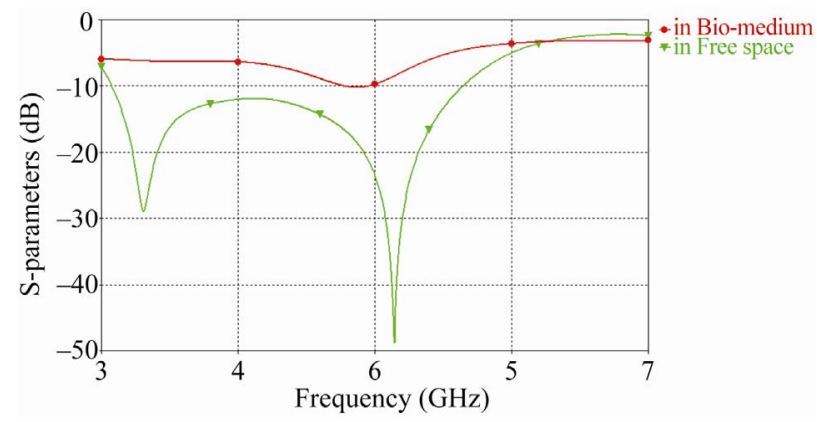

Figure 2. Return loss versus frequency curve of modified DSRDRA.

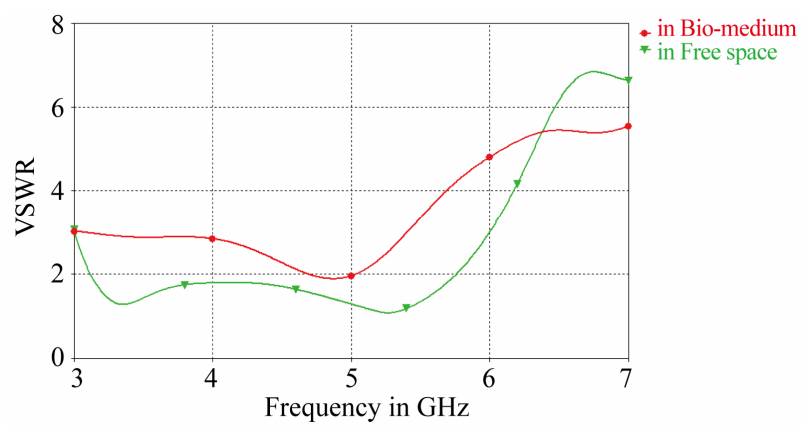

Figure 3. VSWR versus frequency curve of modified DSRDRA. 
can be observed that the values of return loss and input VSWR of the proposed antenna in presence of the synthetic muscle are higher than the corresponding parameter values of antenna in free space. This may be attributed to wave reflections due to abrupt changes in the media when antenna is in the vicinity of the bio-medium.

\subsection{Far Field Performance}

The far field pattern of the proposed antenna in free

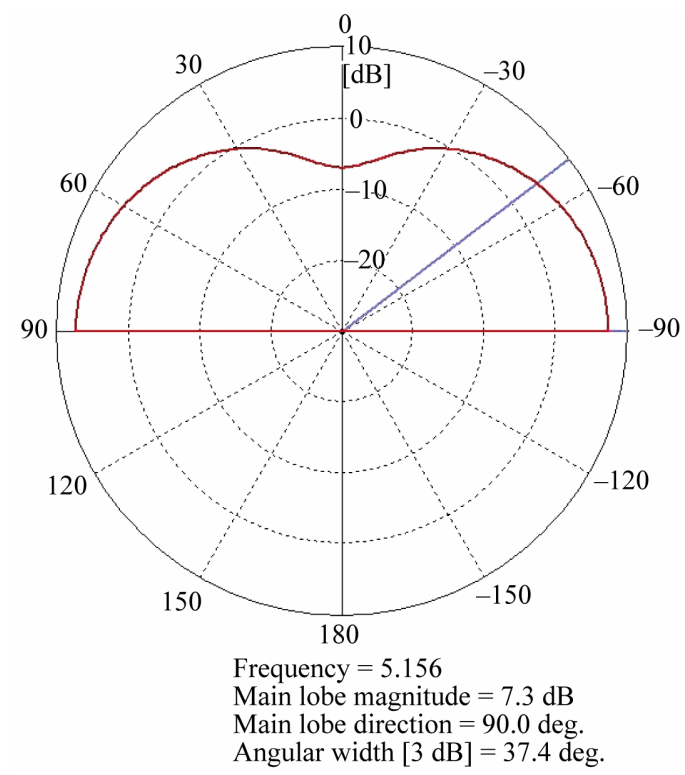

(a)

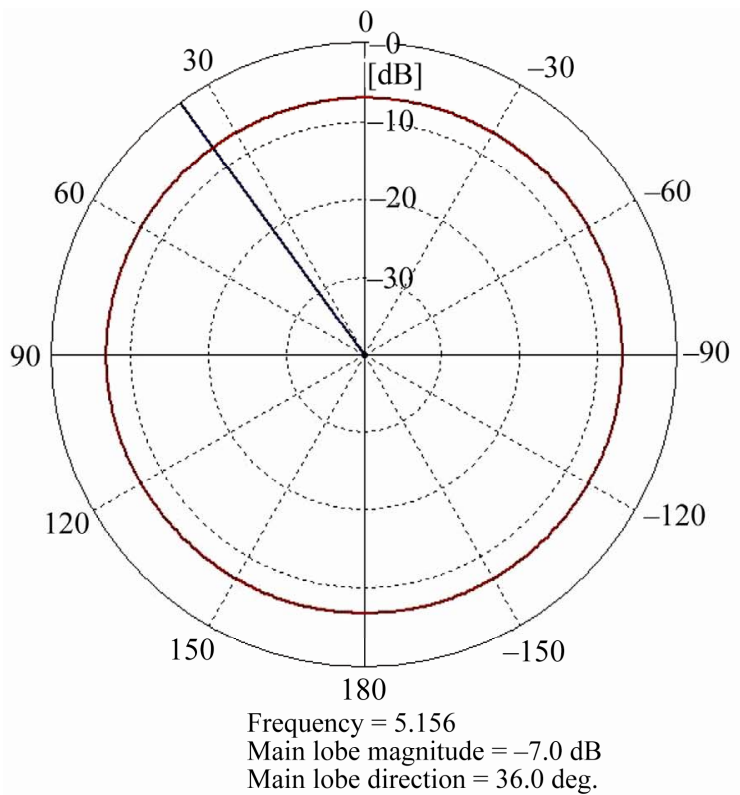

(c) space and in direct contact with phantom muscle layer are studied at $5.156 \mathrm{GHz}$ through simulation using CST Microwave Studio software. The simulated radiation patterns of the proposed antenna in free space and in direct contact with phantom muscle layer at $5.156 \mathrm{GHz}$ are shown in Figures $\mathbf{4}$ and $\mathbf{5}$ respectively. The far field parameters are extracted from Figures $\mathbf{4}$ and $\mathbf{5}$ and the results are shown in Table 1.

From Figure 4 and Table 1 it can be observed that the radiation pattern of the antenna in $x-y$ plane is omni-

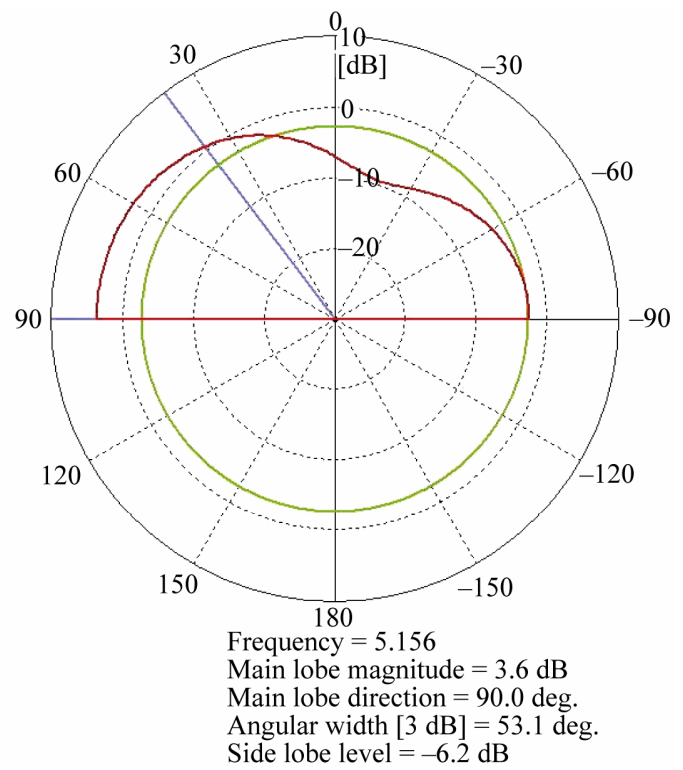

(b)

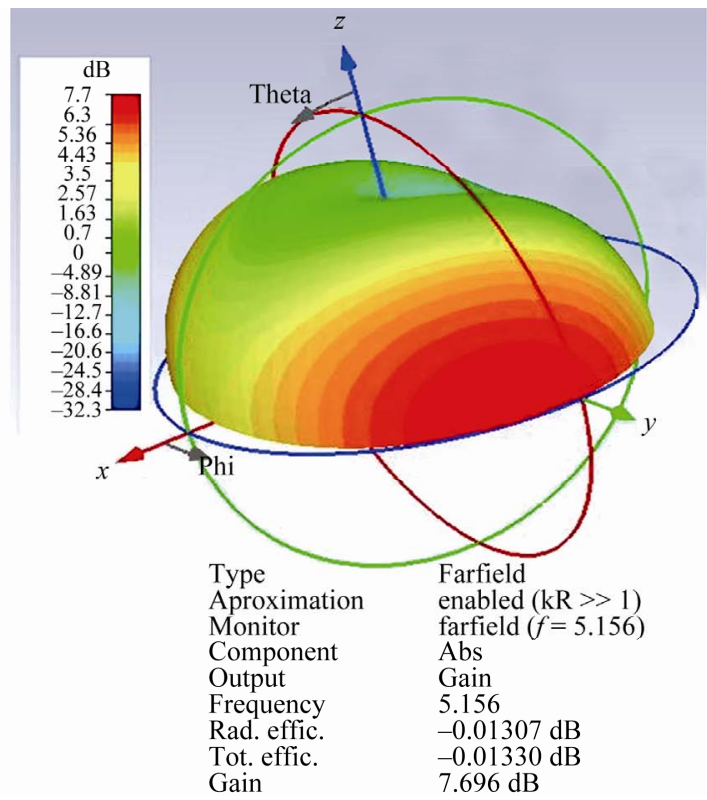

(d)

Figure 4. Far field performance of modified DSRDRA in free space (a) In $x$-z plane; (b) in $y$-z plane; (c) in $x-y$ plane; (d) 3D pattern. 
Farfield 'farfield $[f=5.156]$ [1'] Directivity_Abs [Theta]; Phi $=0.0$ deg.

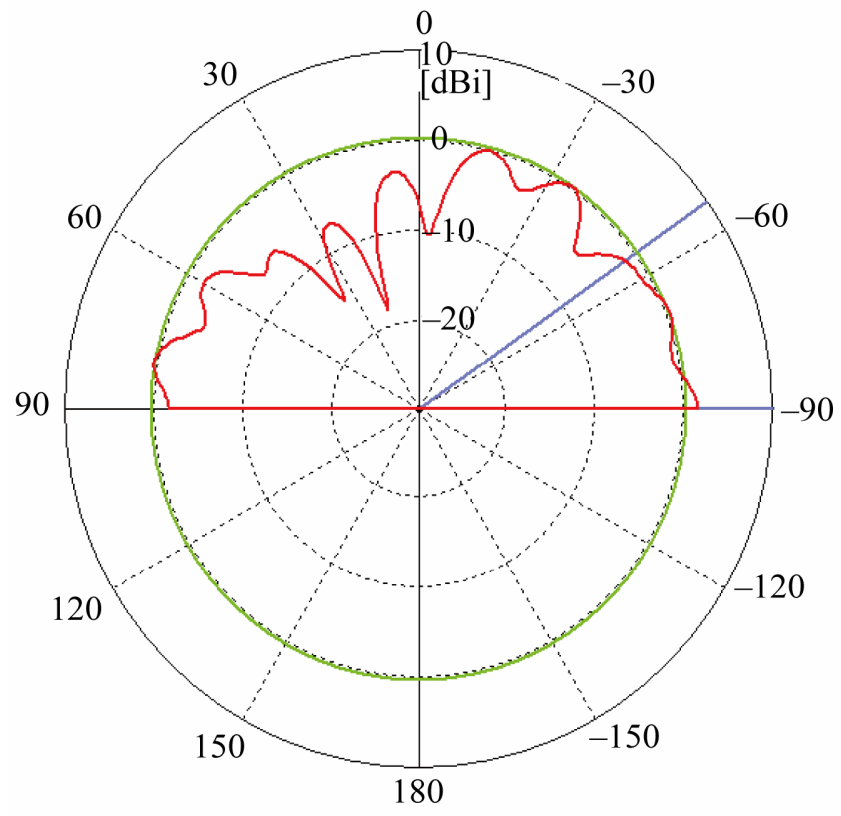

Frequency $=5.156$

Main lobe magnitude $=1.6 \mathrm{dBi}$

Main lobe direction $=90.0 \mathrm{deg}$.

Angular width $[3 \mathrm{~dB}]=35.5 \mathrm{deg}$.

Side lobe level $=-1.2 \mathrm{~dB}$

(a)

Farfield 'farfield $[f=5.156]$ [1'] Directivity_Abs [Phi]; Theta $=0.0 \mathrm{deg}$.

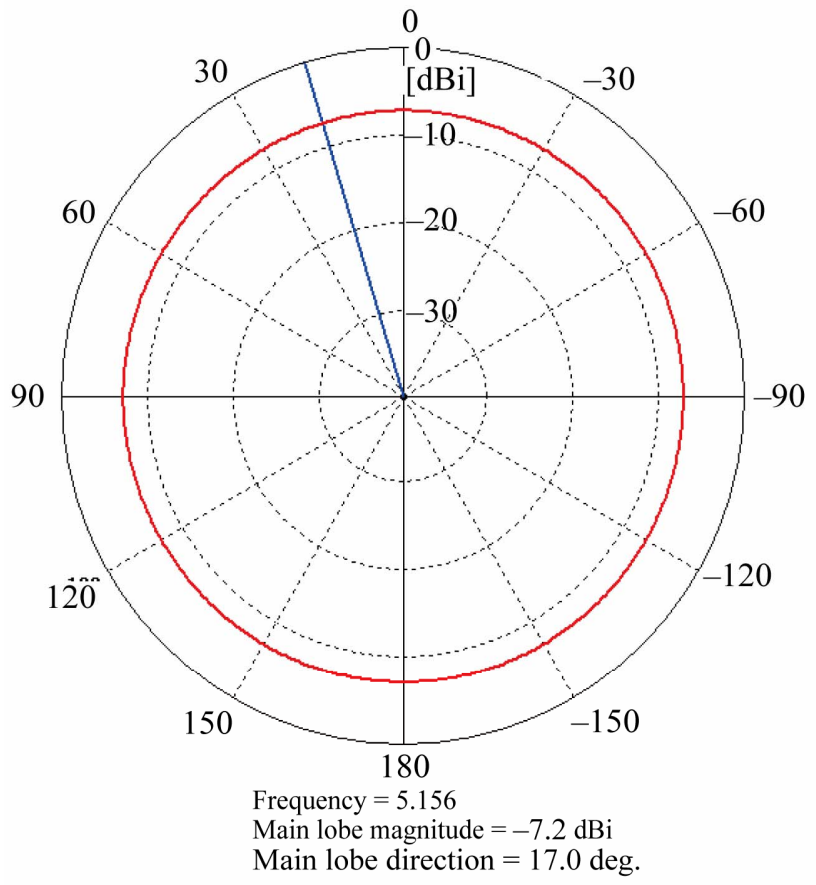

(c)
Farfield 'farfield $[f=5.156]$ [1'] Directivity_Abs [Theta]; Phi $=90.0 \mathrm{deg}$.

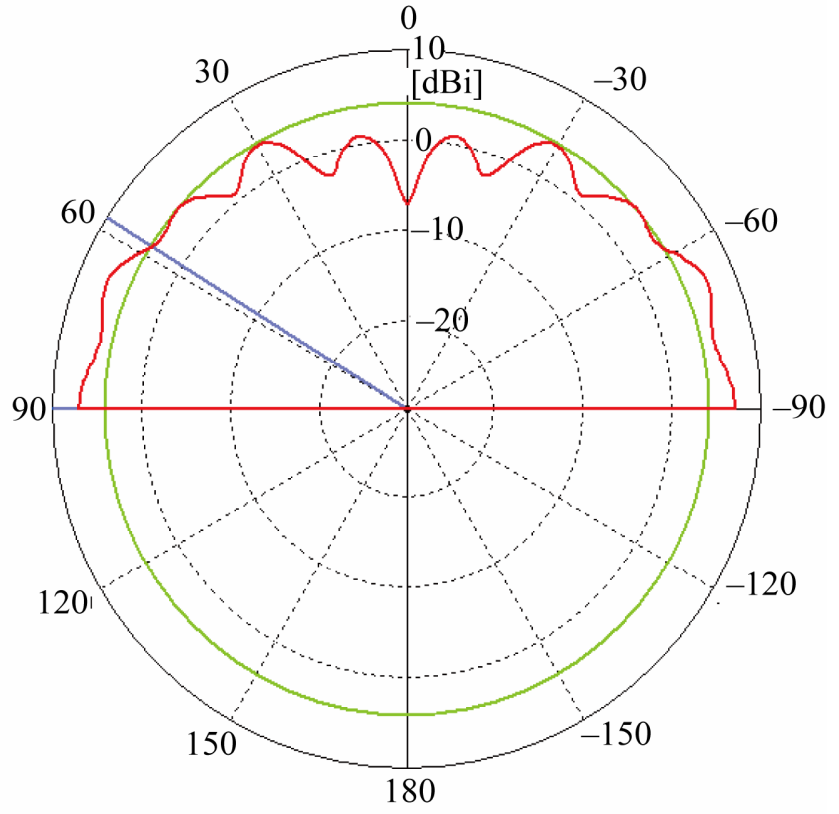

Frequency $=5.156$

Main lobe magnitude $=7.1 \mathrm{dBi}$

Main lobe direction $=90.0 \mathrm{deg}$.

Angular width $[3 \mathrm{~dB}]=32.1 \mathrm{deg}$.

Side lobe level $=-2.8 \mathrm{~dB}$

(b)

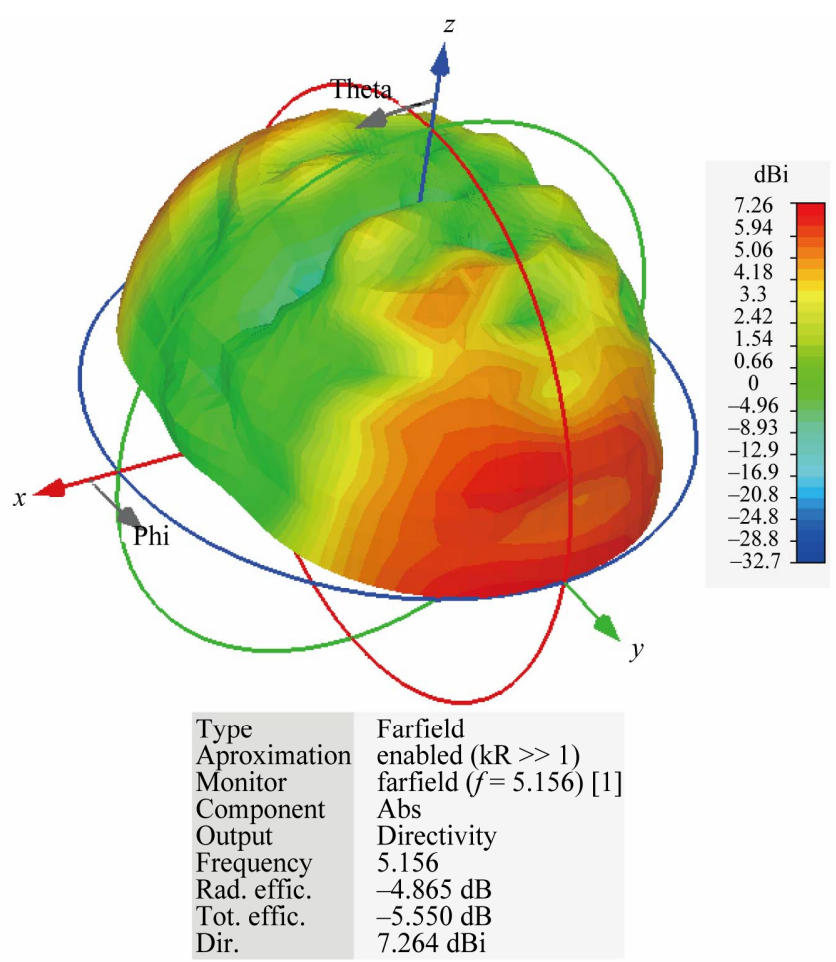

(d)

Figure 5. Far field performance of modified DSRDRA in direct contact with Bio-medium (a) in $x$-z plane; (b) in $y$-z plane; (c) in $x$-y plane; (d) 3D pattern. 
Table 1. Far field parameters of dual segment RDRA.

\begin{tabular}{|c|c|c|c|}
\hline \multicolumn{2}{|c|}{ Far field parameters } & $\begin{array}{c}\text { In Free } \\
\text { space }\end{array}$ & In Bio-medium \\
\hline \multicolumn{2}{|c|}{ Directivity in $\mathrm{dBi}$} & 7.710 & 7.264 \\
\hline \multicolumn{2}{|l|}{ Gain in $\mathrm{dB}$} & 7.696 & 2.399 \\
\hline \multicolumn{2}{|c|}{ Total efficiency in $\%$} & 99.69 & 27.86 \\
\hline \multirow{2}{*}{$\begin{array}{l}\text { 3-dB } \\
\text { Beam-width in } \\
\text { deg. }\end{array}$} & $x$-z Plane & 49.4 & 36.6 \\
\hline & $y$-z Plane & 43.4 & 23.1 \\
\hline \multirow{2}{*}{$\begin{array}{l}\text { Side lobe level } \\
\text { in } \mathrm{dB}\end{array}$} & $x$-z Plane & $\begin{array}{l}\text { No side } \\
\text { lobe }\end{array}$ & -1.2 \\
\hline & $y$-z Plane & -12.0 & -2.8 \\
\hline
\end{tabular}

directional and side lobe is observed in $x$ - $z$ plane. This may be due to presence of metallic plate which significantly reduces the power radiated towards back side of the plate. No side lobes observed in $y$-z plane

From Figure 5 and Table 1 it can be seen that the radiation pattern of the antenna in $x-y$ plane is omni-directional and side lobes are observed in both $x-z$ and $y-z$ planes. This may be attributed to wave reflections due to abrupt changes in the media when antenna is in direct contact with the bio-medium.

From Table 1 it can be seen that when proposed antenna is in direct contact with phantom muscle layer at $5.156 \mathrm{GHz}$, the values of gain, directivity, total efficiency and 3-dB Beam-width of the proposed antenna reduce in comparison to those found for free space.

\subsection{SAR Evaluation}

The origin is selected on the top surface of ground plane coinciding with the central line of dual segment RDRA as shown in Figure 6. The simulation of SAR distri-

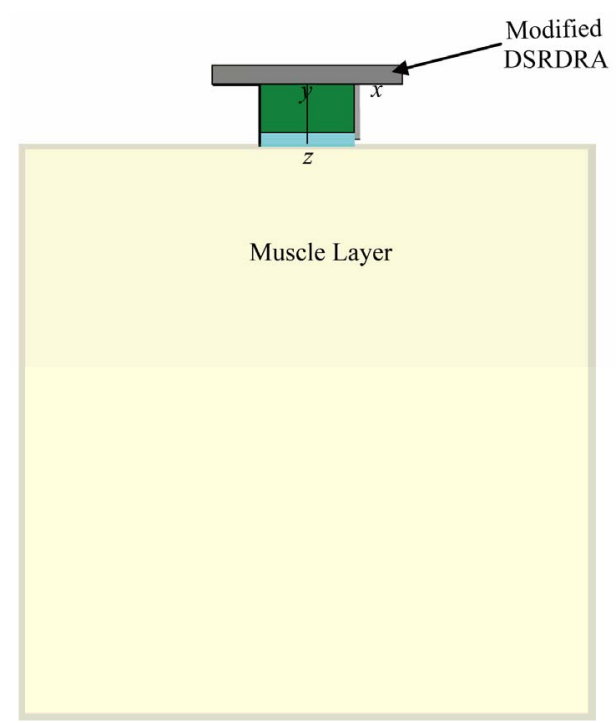

Figure 6. Geometry of modified DSRDRA with muscle layer. butions in a homogenous bio-medium (muscle layer of size $120 \times 120 \times 150 \mathrm{~mm}^{3}$ ), which is separated from the proposed antenna by $0 \mathrm{~mm}, 50 \mathrm{~mm}, 100 \mathrm{~mm}$ and 200 $\mathrm{mm}$ was carried out assuming input power of 0.2 Watt. The simulated SAR $(10 \mathrm{~g})$ distributions in the muscle layer in direct contact with the proposed antenna along $\mathrm{x}$, $\mathrm{y}$ and $\mathrm{z}$ directions at the antenna resonant frequency of $5.165 \mathrm{GHz}$ and at other frequency $3.31 \mathrm{GHz}$ are shown in Figure 7. The SAR (10 g) distributions in the biomedium for the antenna-to-bio-medium separations of 50 $\mathrm{mm}, 100 \mathrm{~mm}$ and $200 \mathrm{~mm}$ at the resonant frequency are shown in Figure 8. The mass density of muscle layer available in literature is $1050 \mathrm{Kg} / \mathrm{m}^{3}$ [14]. The complex permittivity, electrical conductivity of muscle layer

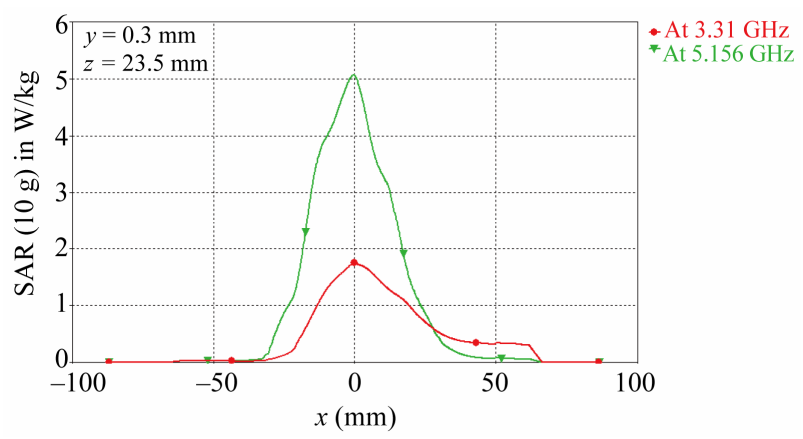

(a)

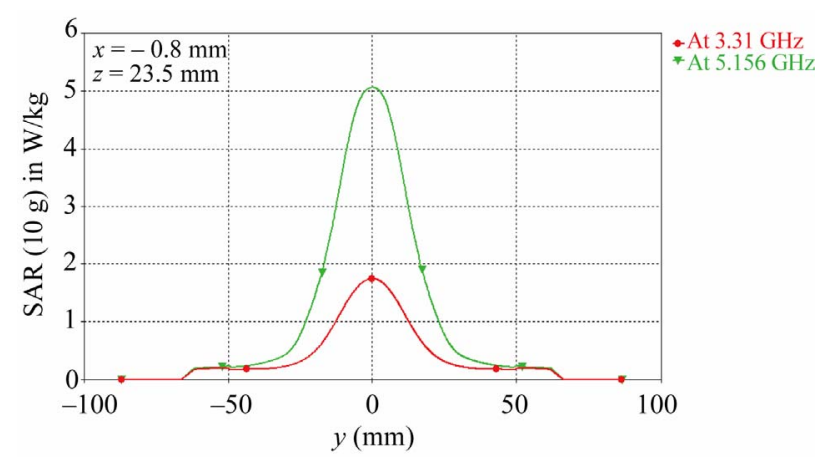

(b)

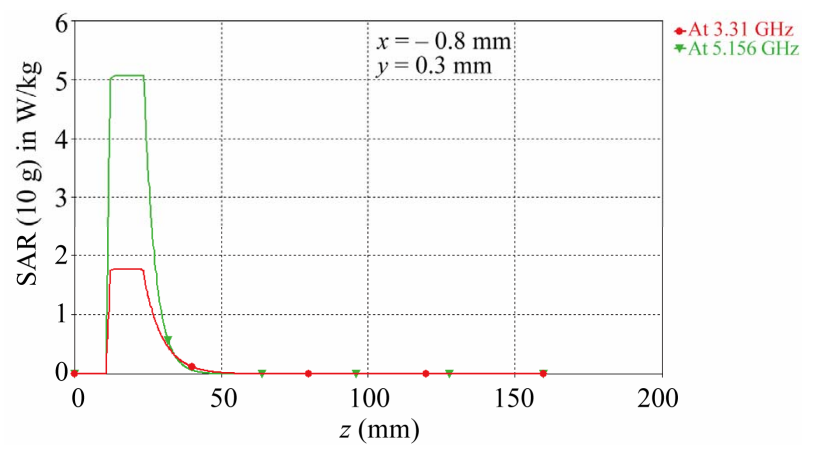

(c)

Figure 7. SAR (10 g) variation in muscle layer due to modified DSRDRA (a) with $x$-axis; (b) with $y$-axis; (c) with $z$-axis. 


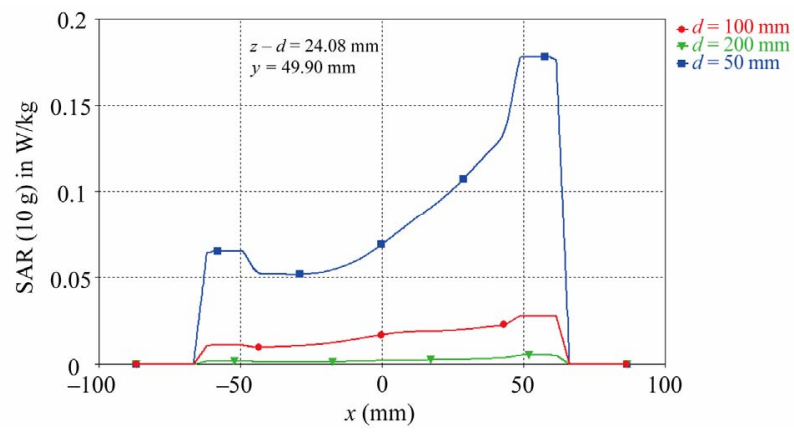

(a)

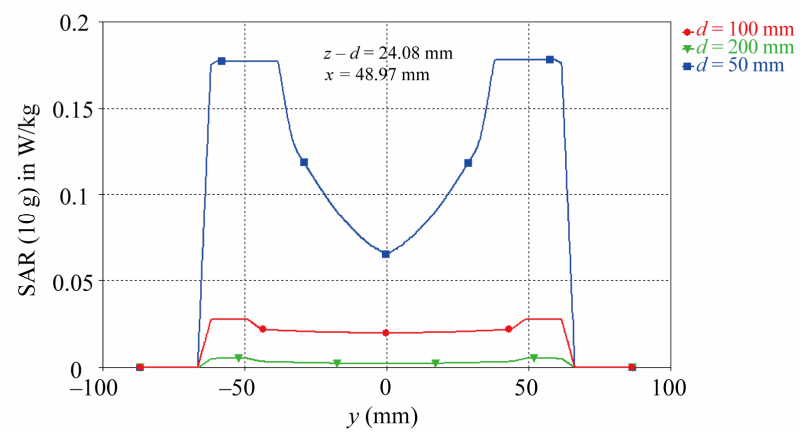

(b)

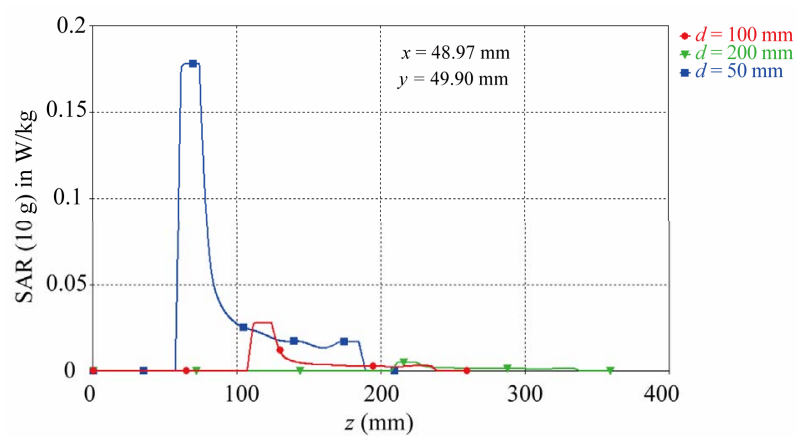

(c)

Figure 8. SAR (10 g) variation in muscle layer due to modified DSRDRA at different antenna-to-bio-medium separations along (a) $x$-axis; (b) $y$-axis; (c) $z$-axis.

compiled from the available literature [15] are 49.399, $4.2156 \mathrm{~S} / \mathrm{m}$ at $5.156 \mathrm{GHz}$ and $51.678,2.3944 \mathrm{~S} / \mathrm{m}$ at 3.31 $\mathrm{GHz}$ respectively.

The three parameters of importance for obtaining the volume of the tissue absorbing significant amount of power are SAR, effective field size (EFS) and penetration depth. The EFS is defined as the area enclosed within the $50 \%$ SAR contour inside the tissue. The penetration depth is the depth at which SAR becomes $1 / \mathrm{e}^{2}$ of its value at the surface [14]. The maximum SAR (10 g), EFS and penetration depth in the bio-medium due to the dual segment RDRA extracted from Figure 7 are shown in Table 2.

From Figure 7 and Table 2, it can be observed that
Table 2. SAR performance of modified DSRDRA in muscle layer.

\begin{tabular}{cccc}
\hline \multicolumn{2}{c}{ Parameters } & At $3.31 \mathrm{GHz}$ & At $5.156 \mathrm{GHz}$ \\
\hline Maximum & $0.2 \mathrm{~W}$ & $1.76519 \mathrm{~W} / \mathrm{kg}$ & $5.05809 \mathrm{~W} / \mathrm{kg}$ \\
SAR $(10 \mathrm{~g})$ & $0.02 \mathrm{~W}$ & $0.176519 \mathrm{~W} / \mathrm{kg}$ & $0.505809 \mathrm{~W} / \mathrm{kg}$ \\
Effective Field Size & $\begin{array}{c}31.6 \times 28.81 \\
\mathrm{~mm}^{2}\end{array}$ & $\begin{array}{c}35.71 \times 28.57 \\
\mathrm{~mm}^{2}\end{array}$ \\
\multicolumn{2}{c}{ Penetration Depth } & $23.03 \mathrm{~mm}$ & $18.28 \mathrm{~mm}$ \\
\hline
\end{tabular}

maximum value of SAR (10 g) increases with frequency and the values of penetration depth reduce with increase in frequency. The trend of changes in EFS and penetration depth in phantom muscle layer may be due to increase in the conductivity of the bio-medium with frequency and frequency dependent characteristics of the proposed antenna. Asymmetry is observed in SAR (10 g) distribution along $x$-direction. This may be due to metallic plate which significantly reduces the power radiated towards back side of the plate and spurious radiation from monopole coaxial probe which is also located along $\mathrm{x}$-axis.

From Figure 8, it can be observed that the value of SAR (10 g) in muscle layer decreases as the separation between muscle layer and antenna increases. This decreasing trend in the value of SAR $(10 \mathrm{~g})$ with increasing antenna-to-bio-medium separation is obvious due to greater beam divergence at larger separation. Also, the shape of SAR (10 g) distribution changes to a great extent with increase in antenna-to-bio-medium separation due to more beam divergence. From Figure 8 the penetration depth for antenna-to-bio-medium separations of $d$ $=50 \mathrm{~mm}, 100 \mathrm{~mm}$ and $200 \mathrm{~mm}$ are found to be 47.11 $\mathrm{mm}, 42.91 \mathrm{~mm}$ and $123.96 \mathrm{~mm}$ respectively. Also, asymmetry is observed in SAR $(10 \mathrm{~g})$ distribution along $\mathrm{x}$-direction. This may be due to metallic plate which significantly reduces the power radiated towards back side of the plate.

The effect of reduction in power input to the antenna from 0.2 to $0.02 \mathrm{~W}$ on maximum SAR ( $10 \mathrm{~g})$ value has also been investigated and the results are presented in Table 2. It is noted from Table 2 that maximum value of SAR (10 g) in muscle layer reduces in proportion to the reduction in power fed to the antenna. The EFS and penetration depth are found to remain unchanged as antenna input power is reduced.

\section{Conclusions}

The simulation studies of SAR distributions in a phantom muscle layer due to modified DSRDRA have been described using CST Microwave Studio simulation software in WiMAX bands. The simulation results of resonant frequency, return loss bandwidth and radiation characteristics of the modified DSRDRA in free space 
are compared with the results for the antenna in direct contact with bio-medium. SAR distributions in a biomedium for different antenna-to-bio-medium separations have been carried out at different frequencies of WiMAX band. From the study it is inferred that the volume of the bio-layer kept near the antenna absorbs significant amount of power in WiMAX band. The value of SAR in the bio-layer increases with frequency as well as with reduction in antenna-to-bio-layer separation. Also, increase in penetration depth and improvement in transverse plane resolution in muscle layer due to the antenna been noticed with reduction in frequency. The results presented here may find potential application in wireless communication field for designing a wideband antenna/wearable antenna and evaluating the power absorption in bio-layers due to the antenna.

\section{References}

[1] M. Okoniewski and M. A. Stuchly, "A Study of the Handset Antenna and Human Body Interaction," IEEE Transactions on Microwave Theory and Techniques, Vol. 44, No. 10, 1996, pp. 1855-1864. doi: $10.1109 / 22.539944$

[2] A. Pradier, D. Lautru, M. F. Wong, F. V. Hanna and J. Wiart, "Rigorous Evolution of Specific Absorption Rate (SAR) Induced in a Multilayer Biological Structure," 2005 European Microwave Conference, Paris, 4-6 October 2005, p. 4. doi:10.1109/EUMC.2005.1610274

[3] R. K. Mongia and P. Bhartia, "DRA-A Review and General Design Relation for Resonant Frequency and Bandwidth," International Journal of Microwave and Millimeter Wave Compute-Aided Engineering, Vol. 4, No. 3, 1994, pp. 230-247.

[4] A. Ittipiboon and R. K. Mongia, "Theoretical and Experimental Investigations on Rectangular Dielectric Resonator Antennas," IEEE Transactions on Antennas and Propagation, Vol. 45, No. 9, 1997, pp. 1348-1356. doi: $10.1109 / 8.623123$

[5] P. Rezaei, M. Hakkak and K. Forooaghi, "Design of Wideband Dielectric Resonator Antenna with a Two Segment Structure," Progress in Electromagnetics Research, Vol. 66, 2006, pp. 111-124. doi:10.2528/PIER06110701
[6] D. Kajfez and A. A. Kishk, "Dielectric Resonator Antenna-Possible Candidate for Adaptive Antenna Arrays," Proceedings of VITEL 2002, International Symposium on Telecommunications, Next Generation Networks and Beyond, Portoroz, 13-14 May 2002.

http://www.ee.olemiss.edu/darko/dra-pcfaaa.pdf

[7] M. Saed and R. Yadla, "Microstrip-Fed Low Profile and Compact Dielectric Resonator Antennas," Progress in Electromagnetics Research, Vol. 56, 2006, pp. 151-162. doi:10.2528/PIER05041401

[8] A. Petosa, "Dielectric Resonator Antennas Handbook," Artech House, London, 2007.

[9] Y. H. Ge and K. P. Esselle, "A Dielectric Resonator Antenna for UWB Applications," IEEE International Symposium on Antennas and Propagation Society, Charleston, 1-5 June 2009, pp. 1-4. doi:10.1109/APS.2009.5171527

[10] S. H. Zainud-Deen, Emad El-Deen, H. A. Sharshar and M. A. Binyamin, "Design of UMTS Dielectric Resonator Antenna for Mobile Phone Including the Biological Effects," National Radio Science Conference (NRSC 2007), Cairo, 13-15 March 2007, pp. 1-8. doi:10.1109/NRSC.2007.371388

[11] T. H. Lee, R. A. Abd-Alhameed and P. S. Excell, "New Dielectric Resonator Antenna Design for Mobile Handsets," International Union for Radio Science URSI 2005. http://rp.iszf.irk.ru/hawk/URSI2005/pdf/BP.10(01068).pdf

[12] V. K. Palikruru, K. Sonoda, R. Surenran and H. Jantunen, "BST-COC Composite Based Rectangular Dielectric Resonator Antenna (DRA) for 2.4-GHz Wrist Applications," Progress in Electromagnetics Research C, Vol. 16, 2010, pp. 195-205. doi:10.2528/PIERC10092104

[13] R. K. Gangwar, S. P. Singh and D. Kumar, "Wideband dual Segment Rectangular Dielectric Resonator Antenna Terminated in a Bio-Medium," 4th International Conference on Communications and Information Technology CIT-2010, Corfu Island, 22-25 July 2010, pp. 64-68.

[14] M. A. Ebrahimi-Ganjeh, "Study of Water Bolus Effect on SAR Penetration Depth and Effective Field Size for Local Hyperthermia," Progress in Electromagnetics Research, Vol. 66, 2006, pp. 111-124.

[15] "An Internet Resource for the Calculation of the Dielectric Properties of Body Tissues in the Frequency Range 10 Hz - 100 GHz," Italian National Research Council, Institute for Applied Physics. http://niremf.ifac.cnr.it/tissprop/ 